\title{
Diffractive Electron-Nucleus Scattering and Ancestry in Branching Random Walks
}

\author{
A. H. Mueller ${ }^{1}$ and S. Munier ${ }^{2, *}$ \\ ${ }^{1}$ Department of Physics, Columbia University, New York, New York 10027, USA \\ ${ }^{2}$ Centre de physique théorique, École polytechnique, CNRS, Université Paris-Saclay, 1 route de Saclay, 91128 Palaiseau, France
}

(Received 25 May 2018; revised manuscript received 3 August 2018; published 23 August 2018)

\begin{abstract}
We point out an analogy between diffractive electron-nucleus scattering events and realizations of onedimensional branching random walks selected according to the height of the genealogical tree of the particles near their boundaries. This correspondence is made transparent in an event-by-event picture of diffraction, emphasizing the statistical properties of gluon evolution, from which new quantitative predictions straightforwardly follow: we are able to determine the distribution of the total invariant mass produced diffractively, which is an interesting observable that can potentially be measured at a future electron-ion collider.
\end{abstract}

DOI: 10.1103/PhysRevLett.121.082001

Introduction.-Diffraction is an elementary consequence of the particle-wave duality postulated by quantum mechanics. Therefore, diffractive patterns are expected to be observed in the scattering of elementary particles off extended objects such as hadrons or nuclei. However, the microscopic interpretation of diffraction turns out to be subtle. Indeed, it is well known that nuclei are loose compounds of hadrons, which themselves appear as fragile bound states of quarks as soon as they are involved in collisions at center-of-mass energies much larger than typically the mass energy of a nucleon. Naively, an energetic electron colliding with a hadron or nucleus, a process known as "deep-inelastic scattering" (DIS), would knock out a quark in each scattering event; then, as a consequence of confinement, the final state would almost systematically consist of many new hadrons distributed all over the detector.

But this is not at all what has been seen experimentally. Indeed, one of the outstanding results of the HadronElectron Ring Accelerator operated at the Deutsches Elektronen-Synchrotron Laboratory until 2007 is the observation of a significant fraction of the events (about 10\%) in which the scattered proton is left intact and is surrounded by an angular region of variable size, empty of particles that we shall call "gap." What has been observed in electronproton collisions should also happen in electron-nucleus scattering. Testing whether this expectation is true can be achieved at a future electron-ion collider.

Diffraction in DIS on protons has been studied extensively, both experimentally (for a review, see [1]) and

Published by the American Physical Society under the terms of the Creative Commons Attribution 4.0 International license. Further distribution of this work must maintain attribution to the author(s) and the published article's title, journal citation, and DOI. Funded by SCOAP. theoretically (see [2] and references therein). But its quantitative theoretical description in the framework of the established theory of the strong interaction, quantum chromodynamics (QCD), remains a challenge. While it is known that the total diffractive cross section can be explained economically and elegantly by saturation models [3], little analytical insight has been gained for more exclusive diffractive observables.

In this Letter, we focus on the diffractive events in deepinelastic scattering off a large nucleus in which the nucleus is left intact, but a hadronic state of large invariant mass $M_{X}$ is nevertheless produced. We explain how to characterize them microscopically, and we show that these hadrons are generated from a similar mechanism as the common ancestor of a set of particles at the frontier of a onedimensional branching random walk. We deduce from this very mechanism a simple analytical prediction, Eq. (4) below, which we test against the numerical integration of a previously known equation governing the energy dependence of high-mass diffraction.

Picture of electron-nucleus scattering at high energy.The scattering of the electron off the nucleus necessarily proceeds through the exchange of a virtual photon $\gamma^{*}$. We shall denote its virtuality by $Q$ and the center-of-mass energy of the $\gamma^{*}$-nucleus subprocess by $W$. These variables are enough to label the total cross section. In the case of diffractive scattering (see Fig. 1), the cross section also depends on the total invariant mass $M_{X}$ of the produced hadrons. It is convenient to use, instead of $M_{X}$, the dimensionless variable $\beta=Q^{2} /\left(Q^{2}+M_{X}^{2}\right)$, in terms of which the DIS experiments traditionally present diffractive data [1], or the logarithm of its inverse $\tilde{y}_{0} \equiv \ln 1 / \beta$. The gap can then be characterized by the Lorentz-invariant rapidity variable $y_{0} \equiv Y-\tilde{y}_{0}$, where $Y \equiv \ln 1 / x_{B j}$, with $x_{B j}=Q^{2} /\left(Q^{2}+W^{2}\right)$, is the total relative rapidity of the photon with respect to the nucleus. 


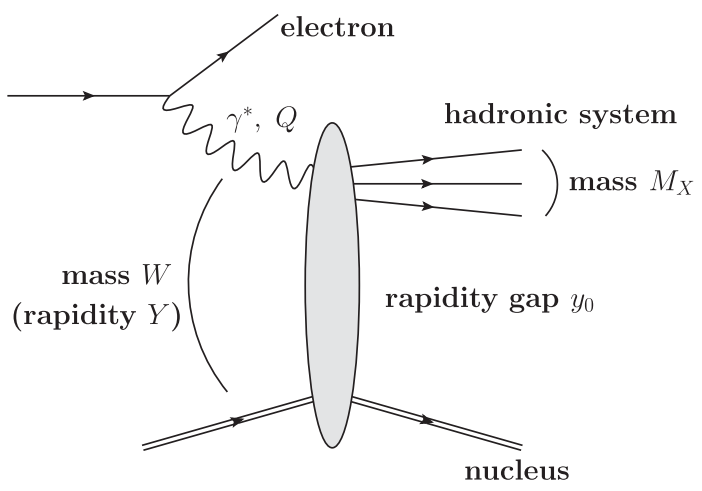

FIG. 1. Schematic representation of a diffractive event. The initial-state particles are incoming from the left, the final state is on the right. The interaction of the electron with the nucleus is mediated by a virtual photon. While the nucleus is transferred unaltered in its nature to the final state, the photon converts to a set of hadrons of total invariant mass $M_{X}$. The rapidity gap is an angular region around the nucleus in which no particle is observed.

When the energy $W$ of the reaction is large, it is possible to choose a reference frame in which the photon is fast enough to almost always convert to a quark-antiquark pair (which we shall call "onium") before interacting. For our purpose, the only relevant parameter to characterize the latter is the distance $r$ between the trajectories of the quarks, which can be considered unchanged throughout a scattering at high relative rapidity. The distribution of $r$ for a given photon virtuality follows from simple electrodynamics. Hence electron-nucleus scattering is tantamount to onium-nucleus scattering. A scattering event occurs as soon as at least one gluon is exchanged between the onium and the nucleus.

A nucleus is a priori a very complicated composite object. However, a large nucleus is made of many hadrons, which can be considered uncorrelated. Considering, furthermore, the number $N_{c}$ of colors to be a large parameter, the rapidity evolution of the forward elastic amplitude $T(r, y)$ for the scattering of the onium off the nucleus can be established within QCD in these limits. It is given by the Balitsky-Kovchegov (BK) equation [4]

$$
\frac{\partial T(r, y)}{\partial y}=\bar{\alpha}[\chi T(r, y)-T \otimes T(r, y)]
$$

where $\bar{\alpha}$ is proportional to the product of the strong coupling constant $\alpha_{s}$ by the number of colors, $\bar{\alpha}=$ $\alpha_{s} N_{c} / \pi ; \chi$ in the first term is the linear operator that acts on a function $f$ of $r$ as

$$
\chi f(r)=\int \frac{d^{2} r^{\prime}}{2 \pi} \frac{r^{2}}{r^{\prime 2}\left(r-r^{\prime}\right)^{2}}\left[f\left(r^{\prime}\right)+f\left(r-r^{\prime}\right)-f(r)\right]
$$

and finally, the second term in the rhs of (1) is the convolution

$$
f \otimes f(r)=\int \frac{d^{2} r^{\prime}}{2 \pi} \frac{r^{2}}{r^{\prime 2}\left(r-r^{\prime}\right)^{2}} f\left(r^{\prime}\right) f\left(r-r^{\prime}\right) .
$$

The elastic onium-nucleus scattering cross section per unit surface [5] is $\sigma_{\mathrm{el}}=T^{2}$ (since $T$ is essentially real at high energy) evaluated at rapidity $y=Y$, and the total cross section is twice $T$ as a consequence of the optical theorem: $\sigma_{\text {tot }}=2 T$. (The total electron-nucleus cross section may then easily be calculated from $\sigma_{\text {tot }}$.) Thanks to these notations, the structure of the BK equation (1) is quite clear. The first term, linear in $T$, encodes the rise of the amplitude due to the multiplication of the gluons in the state of the onium as the rapidity is increased, i.e., as shorterlived quantum fluctuations become relevant for the scattering. It is well known that in the large- $N_{c}$ limit and in a light cone gauge, the Fock state of an onium can conveniently be represented by a set of dipoles of various sizes, and rapidity evolution can be thought of as a cascade of independent $1 \rightarrow 2$ splittings of color dipoles [7]. A light cone perturbation theory calculation in the framework of QCD leads to the expression of the splitting probability density of a dipole of size $r$ into dipoles of sizes $r^{\prime}, r-r^{\prime}$ as its rapidity is increased by $d y$; it reads

$$
d p\left(r \rightarrow r^{\prime}, r-r^{\prime}\right)=\bar{\alpha} d y \frac{d^{2} r^{\prime}}{2 \pi} \frac{r^{2}}{r^{\prime 2}\left(r-r^{\prime}\right)^{2}} .
$$

The operator $\chi$, which is constructed from the integral of $(1 / \bar{\alpha}) d p / d y$, is also the kernel of the evolution equation solved by the mean number density $n(r, y)$ of dipoles at rapidity $y$ in an onium of initial size $r: \partial_{y} n=\bar{\alpha} \chi n$, which is nothing but the Balitsky-Fadin-Kuraev-Lipatov equation [8]. The second term in the BK equation (1), significant only when $T=\mathcal{O}(1)$, keeps the amplitude unitary $(T \leq 1)$ throughout the evolution.

It is useful to note that the BK equation is in essence similar to the nonlinear diffusion equation, known as the Fisher-Kolmogorov-Petrovsky-Piscounov (FKPP) equation [9]: these two equations actually belong to the same universality class [11]. Starting from this correspondence, one can take advantage of the available mathematical knowledge on the FKPP equation (for a review, see Ref. [12]). One knows that, for a vast class of initial conditions, its solution converges to a traveling wave at large $y$, namely, a front connecting $T=1$ for $r$ large to $T=0$ for $r$ small, the rapidity evolution of which consists of a mere translation in $r$. The transition region is located around a rapidity-dependent size $r_{s}(y)$ related to the saturation momentum $Q_{s}$ by $r_{s}=1 / Q_{s}$. The analytical expression of $Q_{s}(y)$ for $\bar{\alpha} y \gg 1$ reads

$$
Q_{s}^{2}(y)=Q_{\mathrm{MV}}^{2} \frac{e^{\bar{\alpha} y \chi^{\prime}\left(\gamma_{0}\right)}}{(\bar{\alpha} y)^{3 / 2 \gamma_{0}}},
$$

up to a multiplicative constant of order one depending on the very definition of $Q_{s}$. The complex function $\chi(\gamma)$ is the 
set of the eigenvalues of the $\chi$ operator associated with its eigenfunctions $r^{2 \gamma}$, and $\gamma_{0}$ solves $\chi\left(\gamma_{0}\right)=\gamma_{0} \chi^{\prime}\left(\gamma_{0}\right)$. Explicitly, $\chi(\gamma)=2 \psi(1)-\psi(\gamma)-\psi(1-\gamma)$, where $\psi$ is the digamma function, and $\gamma_{0} \simeq 0.63$. Equation (2) holds whenever the initial condition falls fast enough as $r$ decreases. More precisely, if $T(r, 0) \underset{r \rightarrow 0}{\sim} r^{2 \lambda}$, then $\lambda$ must be larger than $\gamma_{0}$ [13].

An analytical expression for the asymptotic shape of the front is also known. It reads

$$
T(r, y)=c_{T} \ln \frac{1}{r^{2} Q_{s}^{2}(y)}\left[r^{2} Q_{s}^{2}(y)\right]^{\gamma_{0}},
$$

where $c_{T}$ is a numerical constant. This equation is valid when $T \ll 1$, and in the so-called scaling region [14]. These two conditions translate into the inequalities $1 \ll\left|\ln r^{2} Q_{s}^{2}(y)\right| \ll \sqrt{\chi^{\prime \prime}\left(\gamma_{0}\right) \bar{\alpha} y}$. Throughout, we will always assume that $r$ is such that both these inequalities are fulfilled.

The initial condition for $T$ describes the interaction amplitude of the onium with the nucleus at low energy. A nucleus in its rest frame is a dense system of quarks. In the so-called McLerran-Venugopalan (MV) model [15], it is characterized by a momentum scale $Q_{\mathrm{MV}}$ function of the atomic number. (Its value is of order $1 \mathrm{GeV}$ for heavy nuclei such as lead or gold). The scattering amplitude of an onium with a nucleus may be approximated by $T(r, y=0)=1-e^{-r^{2} Q_{\mathrm{MV}}^{2} / 4}$. Onia of size much larger than typically $r_{\mathrm{MV}} \equiv 1 / Q_{\mathrm{MV}}$ are absorbed, while the nucleus appears transparent to onia of size much smaller than $r_{\mathrm{MV}}$. We note that $T(r, y=0) \sim r^{2}$ for small $r$ : hence, the solutions (2) and (3) indeed apply.

The BK equation (1) is also an equation for the $\tilde{y}$ evolution of the probability $P(r, \tilde{y} \mid R)$ that there be at least a dipole larger than some $R$ in an onium of initial size $r$ [16]. The initial condition in this case reads $P(r, \tilde{y}=0 \mid R)=$ $\theta\left(\ln r^{2} / R^{2}\right)$, which is of course "steep enough" for the asymptotic solution (3) to be valid. Then, thanks to the universality properties of the asymptotic solution to the BK equation, $P(r, \tilde{y} \mid R)$ has the same expression as $T(r, y)$ in Eq. (3) up to the substitutions $y \leftrightarrow \tilde{y}$ and $Q_{\mathrm{MV}} \leftrightarrow 1 / R$ and maybe up to the overall normalization constant. Thus, one can write $\sigma_{\mathrm{tot}} \propto P\left(r, Y \mid 1 / Q_{\mathrm{MV}}\right)$.

Diffraction from rare fluctuations. - If $r$ is small compared to $1 / Q_{s}(Y)$-i.e., the onium is far from the saturation region of the nucleus - then, from Eq. (3), $T$ is small. Since the forward elastic amplitude $T$ is related to the total, elastic, and inelastic cross sections through

$$
\sigma_{\mathrm{tot}}=2 T, \quad \sigma_{\mathrm{el}}=T^{2}, \quad \sigma_{\mathrm{in}}=\sigma_{\mathrm{tot}}-\sigma_{\mathrm{el}},
$$

one sees that $\sigma_{\mathrm{el}}$ is of second order in $T$, while $\sigma_{\text {in }}$ is of first order, and thus dominates $\sigma_{\mathrm{tot}}$.

A diffractive event can occur with non-negligible probability only if a large dipole occurs in the Fock state of the onium at some point in the evolution. Indeed, only for such

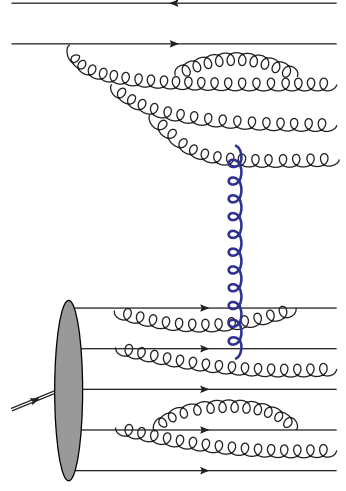

(a)

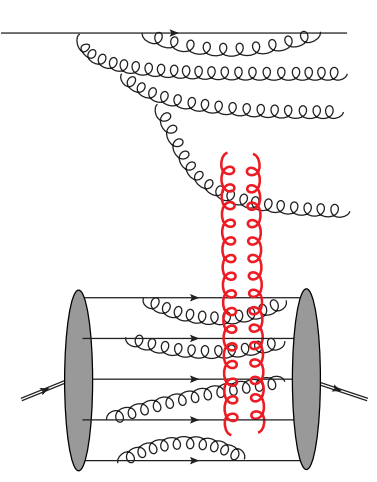

(b)
FIG. 2. Light cone perturbation theory diagrams contributing to onium-nucleus scattering in a frame in which the nucleus carries the rapidity $y_{0}$ and the onium $\tilde{y}_{0}=Y-y_{0}$. (In the large- $N_{c}$ limit, the gluons are replaced by 0 -size $q \bar{q}$ pairs, and the onium Fock state consists of a set of dipoles.) (a) Nondiffractive scattering. (b) Diffractive event with a rapidity gap $y_{0}$. In case (a), the scattering consists of the exchange of [most probably, if $\left.r \ll 1 / Q_{s}(Y)\right]$ one gluon, which results in a breakup of the nucleons and thus of the nucleus. In case (b), one or multiple pairs of gluons grouped in color singlet states are exchanged, in which case the nucleus scatters elastically and no gluon is emitted in the direction of the nucleus momentum.

realizations of the evolution the scattering amplitude can be of order 1, and elastic scattering processes are thus probable. (Examples of Feynman diagrams contributing to the onium-nucleus diffractive vs total amplitudes are shown in Fig. 2.) Assume that such a dipole of size $r_{0}$ is produced at rapidity $\tilde{y}_{0}=Y-y_{0}$. The condition that the whole partonic system scatters elastically with a significant probability is that $r_{0}$ be larger than the inverse saturation scale of the nucleus evaluated at the rapidity $Y-\tilde{y}_{0}=y_{0}$ : $r_{0}>1 / Q_{s}\left(y_{0}\right)$. Such an event will exhibit a rapidity gap of size $y_{0}$.

From this picture, we can immediately infer that the diffractive cross section conditioned to a given rapidity gap $y_{0}$ is tantamount to the probability $P\left(r, \tilde{y}_{0} \mid 1 / Q_{s}\left(y_{0}\right)\right)$. As discussed above, the latter is given by the solution to the BK equation (3) up to the appropriate substitution of the variables and parameters

$$
\frac{d \sigma_{\mathrm{diff}}}{d y_{0}}=c_{\mathrm{diff}} \ln \frac{1}{r^{2} \tilde{Q}_{s}^{2}\left(\tilde{y}_{0}\right)}\left[r^{2} \tilde{Q}_{s}^{2}\left(\tilde{y}_{0}\right)\right]^{\gamma_{0}},
$$

where $c_{\text {diff }}$ is a constant, and the momentum $\tilde{Q}_{s}$ reads

$$
\tilde{Q}_{s}^{2}\left(\tilde{y}_{0}\right)=Q_{s}^{2}\left(y_{0}\right) \frac{e^{\bar{\alpha} \tilde{y}_{0} \chi^{\prime}\left(\gamma_{0}\right)}}{\left(\bar{\alpha} \tilde{y}_{0}\right)^{3 / 2 \gamma_{0}}} .
$$

A straightforward calculation [using Eqs. (2) and (3)] leads to our main result for the distribution of the size of the rapidity gap. The simplest expression is obtained for the 


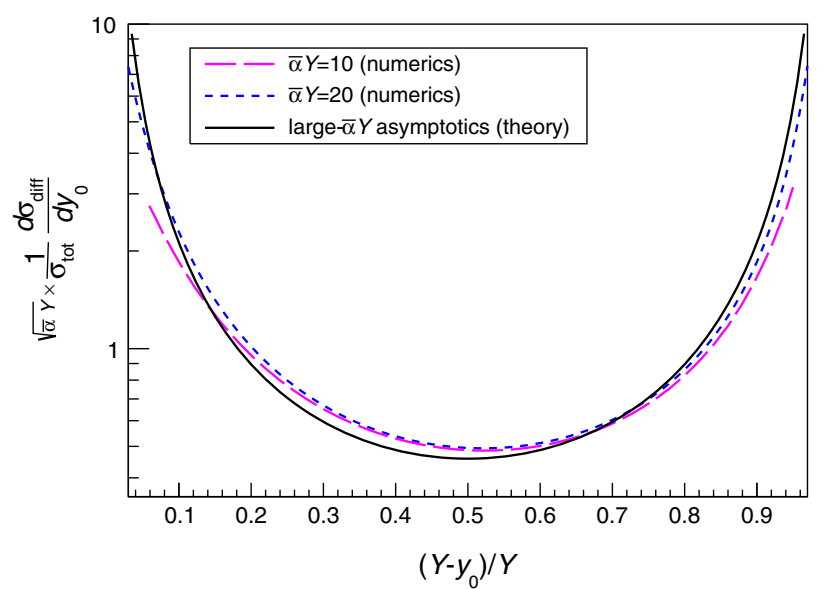

FIG. 3. Rescaled distribution of $\left(Y-y_{0}\right) / Y$ calculated from the numerical integration of the KL equation for two different values of the total rapidity, compared to the asymptotic theoretical formula (4), with an ad hoc global normalization factor. The onium size is chosen to be well in the scaling region. (See the main text for the details).

differential diffractive cross section normalized by the total cross section

$$
\frac{1}{\sigma_{\text {tot }}} \frac{d \sigma_{\text {diff }}}{d y_{0}} \propto\left(\frac{\bar{\alpha} Y}{\bar{\alpha} y_{0} \bar{\alpha}\left(Y-y_{0}\right)}\right)^{3 / 2}
$$

in such a way that the overall coefficient, which we have not been able to determine, is a pure number independent of the parameters $r$ and $Y$. The formula (4) is valid whenever $\bar{\alpha} y_{0}$ is distant from its two boundaries at zero and $\bar{\alpha} Y$ by more than typically one unit. We also recall that this result is an asymptotic limit for $\bar{\alpha} Y$ large and that it holds when the size $r$ of the onium is picked in the scaling region.

Genealogy in branching random walks.-Our whole discussion of the structure of diffractive events turns out to be parallel to the discussion of the genealogy of particles near the boundary of a branching random walk (BRW) in Ref. [17].

Consider a BRW in time $t$ and in the real variable $x$, starting with one single particle, defined with the help of a stochastic process such that the mean density of particles $n(x, t)$ obeys the equation $\partial_{t} n=\chi n ; \chi$ is an appropriate operator acting on $n$ viewed as a function of $x$ and encoding the microscopic process: e.g., $\chi=\partial_{x}^{2}+1 . \chi$ admits the eigenfunctions $e^{-\gamma x}$ and we denote by $\chi(\gamma)$ the corresponding eigenvalues. After the (large) time $t$, pick exactly two particles, choosing them either (i) according to the Boltzmann weight $e^{-\lambda x}$ (i.e., the particle number $j$ sitting at position $x_{j}$ at time $t$ is picked with probability $e^{-\lambda x_{j}} / \sum_{k} e^{-\lambda x_{k}}$ ) or (ii) to be exactly the two leftmost particles, and look for the first common ancestor splitting time $t-t_{0}$. Then, according to Ref. [17], $t_{0}$ is distributed as

$$
p\left(t_{0}\right)=c_{p}\left(\frac{t}{t_{0}\left(t-t_{0}\right)}\right)^{3 / 2}, \quad \text { with } \quad c_{p}=\frac{1}{\bar{\gamma}} \frac{1}{\sqrt{2 \pi \chi^{\prime \prime}\left(\gamma_{0}\right)}},
$$

where $\bar{\gamma}=\lambda$ in case (i) if $\lambda>\gamma_{0}$, and $\bar{\gamma}=\gamma_{0}$ in case (ii). $\gamma_{0}$ solves $\chi\left(\gamma_{0}\right)=\gamma_{0} \chi^{\prime}\left(\gamma_{0}\right)$.

In the same way as in our diffraction calculation, the common ancestor of the boundary particles also corresponds to a fluctuation, in the form of a particle sent to the left of the expected position of the leftmost particle, occurring in the course of the evolution at time $t-t_{0}$. Hence, the two problems are intimately related: up to the overall normalization, which is determined in the case of the genealogies, but not in the case of diffraction, $\left(1 / \sigma_{\text {tot }}\right)\left(d \sigma_{\text {diff }} / d y_{0}\right)$ corresponds to $p\left(t_{0}\right)$, with the identifications $\bar{\alpha} Y \leftrightarrow t, \bar{\alpha} y_{0} \leftrightarrow t_{0}$.

Numerical test.-An equation for the diffractive cross section with a rapidity gap $y_{0}$ was established some time ago in QCD by Kovchegov and Levin (KL) [18] (see also Refs. [2,19]). It can be put in the form of two appropriately matched evolution equations in the total rapidity variable $y$, which both turn out to be of the BK type. While this formulation has not led to much analytical insight, in particular, for the gap distribution we are addressing here, it is very convenient for the numerical computation of the diffractive cross section, since the BK equation is easily discretized, implemented, and solved using standard algorithms [20].

We have computed the rapidity-gap distribution for two values of the total rapidity, $\bar{\alpha} Y=10$ and 20. (These rapidities are of course too large to be realistic for phenomenology, but our goal here is to test our asymptotic prediction.) We have chosen $r$ in such a way that $\left|\ln r^{2} Q_{s}^{2}(Y)\right| \simeq 7.2$, comfortably in the scaling region in both cases. The results are presented in Fig. 3 and compared to the analytical prediction (4) in appropriately rescaled variables chosen such that the expected asymptotic distribution be independent of $\bar{\alpha} Y$. The overall coefficient of the latter is not predicted in our approach. We could fit it to the numerical data, but interestingly enough, just setting it to be that predicted for BRW, namely, $c_{p}$ in Eq. (5), with $\bar{\gamma}=1$, leads to a remarkably good agreement between the numerical data and the prediction.

Conclusion.-We have found that the distribution of the size $y_{0}$ of rapidity gaps in diffractive onium-nucleus scattering can be calculated analytically for fixed largecenter-of-mass energies. Surprisingly enough, our quantitative prediction follows quite straightforwardly from simple considerations on the mechanism how the Fock state of a quark-antiquark pair evolves when one increases its rapidity. The essence of this evolution is that of a onedimensional branching random walk, and viewed in such a picture, diffractive events are due to the existence of a large fluctuation in the evolution. The rapidity at which it occurs determines the size of the gap. 
This large fluctuation can also be identified with the common ancestor of a few extreme objects generated by the BRW. The latter problem is of interest in the study of disordered systems. It was known before that the BK and FKPP equations are in the same universality class [10], and also that the energy evolution of the scattering amplitude of ultrahigh-energy hadrons may be analogous to the time evolution of a reaction-diffusion process, the evolution of which is described by an equation belonging to the universality class of the stochastic FKPP equation [21]. But to our knowledge, this is the first time that the statistical properties of genealogical trees prove of direct relevance in the context of particle or nuclear physics. Hence, our Letter contributes to bridge a priori unrelated fields of physics.

The results we have obtained here can be converted into new predictions for the mass distribution in diffractive virtual photon-nucleus scattering $\beta d \sigma_{\text {diff }}^{\gamma^{*} A} / d \beta$, measurable at a future electron-ion collider. At fixed $W$ and $Q$, the latter is actually identical to the distribution of rapidity gaps $d \sigma_{\text {diff }}^{\gamma^{*} A} / d y_{0}$, which can be calculated by convoluting the onium cross section $d \sigma_{\text {diff }} / d y_{0}$ with the known distribution of the sizes $r$ of quark-antiquark pairs in the Fock state of the virtual photon (see, e.g., [3]). This is actually straightforward when the photon is polarized longitudinally, since in this case, the distribution is peaked around the inverse photon virtuality; i.e., $r$ can essentially be identified to $1 / Q$. For transversely polarized photons, since the $r$ distribution corresponding to a given $Q$ is wider, a better knowledge of $d \sigma_{\text {diff }} / d y_{0}$ outside of the scaling region would be needed. Further developments, along with more numerical studies, can be found in Ref. [22].

The work of A.H. M. is supported in part by the U.S. Department of Energy Award No. DE-FG02-92ER40699. The work of S.M. is supported in part by the Agence Nationale de la Recherche under Project No. ANR-16CE31-0019. We thank Bernard Pire for urging us to try to make the material of the present Letter accessible to a wider audience and for useful suggestions on the manuscript.

*Corresponding author. stephane.munier@polytechnique.edu

[1] L. Schoeffel, Prog. Part. Nucl. Phys. 65, 9 (2010).

[2] Y. V. Kovchegov and E. Levin, Quantum Chromodynamics at High Energy (Cambridge University Press, Cambridge, England, 2012), Vol. 33.

[3] K. J. Golec-Biernat and M. Wusthoff, Phys. Rev. D 59, 014017 (1998).

[4] I. Balitsky, Nucl. Phys. B463, 99 (1996); Y. V. Kovchegov, Phys. Rev. D 61, 074018 (2000).
[5] Throughout, we focus on the diffractive cross section per unit surface in the transverse plane, thus evaluated at a fixed impact parameter $b$. To obtain the cross section integrated over $b$, one would need a model for the $b$ dependence of $Q_{\mathrm{MV}}$ (see, e.g., [6] in the case of the proton). One may also simply assume $Q_{\mathrm{MV}}$ constant over the surface of the nucleus, as was done for DIS off the proton in Ref. [3], in which case the integration over $b$ just amounts to multiplying by the cross section surface of the nucleus.

[6] H. Kowalski and D. Teaney, Phys. Rev. D 68, 114005 (2003); H. Kowalski, L. Motyka, and G. Watt, Phys. Rev. D 74, 074016 (2006); G. Watt and H. Kowalski, Phys. Rev. D 78, 014016 (2008).

[7] A. H. Mueller, Nucl. Phys. B415, 373 (1994).

[8] L. N. Lipatov, Yad. Fiz. 23, 642 (1976) [Sov. J. Nucl. Phys. 23, 338 (1976)]; E. A. Kuraev, L. N. Lipatov, and V. S. Fadin, Zh. Eksp. Teor. Fiz. 72, 377 (1977) [Sov. Phys. JETP 45, 199 (1977)]; I. I. Balitsky and L. N. Lipatov, Yad. Fiz. 28, 1597 (1978) [Sov. J. Nucl. Phys. 28, 822 (1978)].

[9] R. A. Fisher, Ann. Eugenics 7, 355 (1937); A. Kolmogorov, I. Petrovsky, and N. Piscounov, Bull. Univ. État Moscou A 1, 1 (1937).

[10] S. Munier and R. B. Peschanski, Phys. Rev. Lett. 91, 232001 (2003).

[11] An exact mapping can be exhibited for the Fourier transform of $T$ defined as $\tilde{T}(k, y)=\int d^{2} r /\left(2 \pi r^{2}\right) e^{i k r} T(r, y)$ and in the so-called diffusive approximation; see Ref. [10].

[12] W. van Saarloos, Phys. Rep. 386, 29 (2003).

[13] M. Bramson, Mem. Am. Math. Soc. 44, iv+190 (1983).

[14] A. M. Stasto, K. J. Golec-Biernat, and J. Kwiecinski, Phys. Rev. Lett. 86, 596 (2001); E. Iancu, K. Itakura, and L. McLerran, Nucl. Phys. A708, 327 (2002); A. H. Mueller and D. N. Triantafyllopoulos, Nucl. Phys. B640, 331 (2002).

[15] L. D. McLerran and R. Venugopalan, Phys. Rev. D 49, 2233 (1994).

[16] A. H. Mueller and S. Munier, Phys. Lett. B 737, 303 (2014).

[17] B. Derrida and P. Mottishaw, Europhys. Lett. 115, 40005 (2016).

[18] Y. V. Kovchegov and E. Levin, Nucl. Phys. B577, 221 (2000).

[19] A. Kovner and U. A. Wiedemann, Phys. Rev. D 64, 114002 (2001); M. Hentschinski, H. Weigert, and A. Schafer, Phys. Rev. D 73, 051501 (2006); Y. Hatta, E. Iancu, C. Marquet, G. Soyez, and D. N. Triantafyllopoulos, Nucl. Phys. A773, 95 (2006).

[20] E. Levin and M. Lublinsky, Phys. Lett. B 521, 233 (2001); Eur. Phys. J. C 22, 647 (2002); Nucl. Phys. A712, 95 (2002).

[21] E. Iancu, A. H. Mueller, and S. Munier, Phys. Lett. B 606, 342 (2005); E. Iancu and D. N. Triantafyllopoulos, Nucl. Phys. A756, 419 (2005).

[22] A. H. Mueller and S. Munier, companion paper, Phys. Rev. D 98, 034021 (2018). 